\title{
EDUCAÇÃO EM SAÚDE COMO ESTRATÉGIA DE QUALIFICAÇÃO DA ASSISTÊNCIA ÀS GESTANTES NO INTERIOR DO CEARÁ
}

\section{HEALTH EDUCATION AS A STRATEGY FOR QUALIFICATION OF ASSISTANCE TO PREGNANT WOMEN IN CEARÁ}

\section{EDUCACIÓN EN SALUD COMO ESTRATEGIA DE CALIFICACIÓN DE LA ASISTENCIA A LOS GESTANTES EN EL INTERIOR DEL CEARÁ}

Maria Girlane Sousa Albuquerque Brandão ${ }^{1}$, Sibele Pontes Rocha ${ }^{2}$, Ana Suelen Pedroza Cavalcante ${ }^{3}$, Maria Amélia Carneiro Bezerra ${ }^{4}$, Maristela Inês Osawa Vasconcelos ${ }^{5}$

\section{RESUMO}

Objetivo: relatar a experiência de ações de educação em saúde com grupo de gestantes. Método: relato de experiência de ações com gestantes em Centro de Saúde da Família do interior do Ceará, mediante as vivências práticas do módulo Internato I da grade curricular do curso de Enfermagem de Universidade Pública Cearense. Como estratégia de adesão, antes dos encontros havia a entrega de convites no domicilio por Agentes Comunitários de Saúde. A abordagem grupal aplicada fundamentou-se no Círculo de Cultura. Resultados: foram desenvolvidos dez encontros de intervenção observando as necessidades das gestantes, com apoio da enfermeira e Programa de Residência Multiprofissional em Saúde da Família, com troca de saberes, experiências, esclarecimento de dúvidas entre acadêmica, equipe de saúde da família e gestantes, com vistas à promoção da saúde. Considerações Finais: As vivências educativas propiciaram encontros participativos e dialogados com fortalecimento de vínculos entre os diferentes atores envolvidos no processo de gestação.

Descritores: Educação em Saúde; Gravidez; Estratégia Saúde da Família; Enfermagem.

\section{ABSTRACT}

Objective: to report the experience of health education actions with a group of pregnant women. Method: report of experience of actions with pregnant women in the Family Health Center of the interior of Ceará, through the practical experiences of the Internship I module of the curriculum of the Nursing course of the Public University of Ceará. As a strategy for adherence, before the meetings there were the delivery of invitations at home by Community Health Agents. The applied group approach was based on the Culture Circle. Results: Ten intervention meetings were carried out, observing the needs of the pregnant women, with the support of the nurse and Multiprofessional Residency Program in Family Health, with exchange of knowledge, experiences, clarification of doubts between academic, family health team and pregnant women

\footnotetext{
${ }^{1}$ Mestranda em Enfermagem pela Universidade da Integração Internacional da Lusofonia Afro-Brasileira (UNILAB). ${ }^{2}$ Mestranda em Saúde da Família pela Universidade Federal do Ceará (UFC).

${ }^{3}$ Doutoranda em Saúde Coletiva pela Universidade Estadual do Ceará (UECE).

${ }^{4}$ Doutora em Farmacologia. Docente da Universidade Estadual Vale do Acaraú (UVA).

${ }^{5}$ Doutora em Enfermagem. Pós-doutorado em Cuidados Clínicos em Enfermagem e Saúde. Docente da Universidade Estadual Vale do Acaraú (UVA).
} 
with a perspective to health promotion. Conclusion: The educational experiences provided participatory and dialogued meetings with the strengthening of ties between the different actors involved in the gestation process.

Descriptors: Health Education, Pregnancy, Family Health Strategy, Nursing.

\section{RESUMEN}

Objetivo: relatar la experiencia de acciones de educación en salud con grupo de gestantes. Método: relato de experiencia de acciones con gestantes en Centro de Salud de la Familia del interior de Ceará, mediante las vivencias prácticas del módulo Internado I de la parrilla curricular del curso de Enfermería de Universidad Pública Cearense. Como estrategia de adhesión, antes de los encuentros había la entrega de invitaciones a domicilio por Agentes Comunitarios de Salud. El enfoque grupal aplicado se fundó en el Círculo de Cultura. Resultados: se desarrollaron diez encuentros de intervención observando las necesidades de las gestantes, con apoyo de la enfermera y Programa de Residencia Multiprofesional en Salud de la Familia, con intercambio de saberes, experiencias, aclaración de dudas entre académica, equipo de salud de la familia y gestantes, con para la promoción de la salud. Consideraciones Finales: Las vivencias educativas propiciaron encuentros participativos y dialogados con fortalecimiento de vínculos entre los diferentes actores involucrados en el proceso de gestación.

Descriptores: Educación en Salud; Embarazo; Estrategia Salud de la Familia; Enfermería.

\section{INTRODUÇÃO}

A assistência à mulher na gestação propõe a unidade básica de saúde (UBS) como porta de entrada preferencial ao sistema de saúde, ponto de atenção estratégico para o acompanhamento continuado da gravidez, e elege a Estratégia Saúde da Família (ESF) como proposta programática principal para a reorganização dos modelos e práticas no âmbito primário de atenção. ${ }^{1}$

A gravidez é uma experiência complexa, com aspectos diferentes para cada mulher. Além da dimensão biológica, é um processo social que envolve o coletivo e que mobiliza a família e o meio no qual a mulher está inserida. Neste contexto, a assistência pré-natal consiste no acompanhamento da gestante, que se configura como momento de aprendizagem para a mulher e sua familia, e que pode permitir a detecção de anormalidades materno-fetal, destacando-se como um fator essencial na proteção e na prevenção a eventos adversos a saúde obstétrica. $^{2}$

Durante o pré-natal, é interessante a criação de um espaço de educação em saúde, a fim de preparar a mulher para viver a gestação e o parto de forma positiva, integradora, enriquecedora e feliz. $\mathrm{O}$ processo educativo é fundamental não só para a aquisição de conhecimentos sobre o processo de gestar e parir, mas também para o seu fortalecimento como ser e cidadã. ${ }^{3}$ 
Educação em saúde não se resume apenas a processos de intervenção na doença, mas é um processo permanente de ensino e aprendizagem que busca superar a compreensão de saúde somente como o contrário de doença, relacionando-a, desse modo, à qualidade de vida. ${ }^{4}$

abordagem grupal, por sua vez, propicia um ambiente ideal para a promoção da saúde, pelo processo de ensinar-aprender. A gestação é um período importante para realização de ações educativas, por propiciar um intercâmbio de vivências e conhecimentos. 5 Uma das estratégias de educação em saúde que pode ser utilizada nestes espaços é o Círculo de Cultura, um método de Paulo Freire, que é capaz de estabelecer o diálogo, a participação, o respeito, trabalho em grupo, discussão, troca de experiências e vivências, ensinoaprendizado mútuo sobre diversos temas e capacitar as pessoas para refletir sobre sua realidade. $^{6} \quad \mathrm{O}$ módulo de Internato I da matriz curricular do curso de enfermagem da Universidade Estadual Vale do Acaraú (UVA) oferece uma imersão na Atenção Primária à Saúde, com desenvolvimento de uma intervenção a partir da realidade observada, permeando a melhoria das condições de vida da comunidade, excitando a extensão que a universidade propõe. $\quad$ Em face das vivências procedentes desse módulo, evidenciou-se que o grupo de gestantes de um determinado Centro de Saúde da Familia (CSF) no interior do Ceará estava temporariamente desativado e, ao observar a importância do retorno do grupo, optou-se por realizar da reativação do mesmo.

O relato justifica-se por apresentar ações de educação em saúde, com atuação da equipe multiprofissional, caracterizandose como estratégia de promoção da saúde e assistência às gestantes, além de contribuir no processo de formação e qualificação profissional de acadêmicos e da equipe da Atenção Básica, com fortalecimento das práticas educativas dialogadas e participativas.

Esse trabalho tem por objetivo relatar a experiência de ações de educação em saúde, utilizando o Círculo de Cultura de Paulo Freire com um grupo de gestantes.

\section{MÉTODO}

Trata-se de um estudo descritivo, qualitativo, do tipo relato de experiência, sobre intervenções vivenciadas em um CSF no interior do Ceará. As vivências ocorreram durante o Internato I, módulo que integra a grade curricular do curso de Enfermagem da UVA, no período de julho a novembro de 
2017.

A partir das vivências práticas do Módulo Internato I, foi possível conhecer a realidade do CSF e identificar que o grupo de gestantes estava desativado. Ao reconhecer o valor da educação em saúde durante a gestação, elegeu-se a reativação do grupo para ser tema da intervenção na comunidade.

Para o sucesso da reativação, foram utilizadas estratégias para que as gestantes se sentissem mais acolhidas e importantes no grupo. Neste sentido, antes de cada encontro, elas recebiam um convite com data, horário e temática, entregues pelos Agentes Comunitários de Saúde (ACS) em domicilio, e este convite era sempre reforçado durante as consultas de pré - natal.+

A abordagem grupal aplicada nos encontros se fundamentou no Círculo de Cultura de Paulo Freire que incentiva a realização de encontro entre as pessoas ou grupos de pessoas que se dedicam ao trabalho didático-pedagógico e outras vivências culturais e educacionais, pautadas no processo de ensino e de aprendizagem, ${ }^{6}$ tendo como coordenadores a interna de enfermagem, enfermeira do CSF, Agentes Comunitários de Saúde e residentes multiprofissionais em Saúde da Familia.

A periodicidade e temas dos encontros foram determinados em consenso com as gestantes, ficando estabelecidos encontros semanais. Todos os encontros foram realizados em quatro momentos: acolhimento, explanação da temática, avaliação do momento e escolha do próximo tema.

Vale ressaltar que o estudo não necessitou da aprovação do CEP, mas foi aceito e aprovado pela gerência da Unidade Básica de Saúde, sendo respeitados todos os preceitos da Resolução 466/12, do Conselho Nacional de Saúde.

\section{APRESENTAÇÃO DA EXPERIÊNCIA}

Durante o período da intervenção aconteceram 10 encontros, nos quais foi possível a discussão de temáticas de acordo com os interesses das participantes. $\mathrm{O}$ primeiro encontro foi realizado com a participação da interna de enfermagem, enfermeira da unidade, três ACS e quatro gestantes, com apresentação da temática de autoestima positiva na gestação. Realizouse a dinâmica do espelho, em que cada gestante recebeu uma caixa com um espelho dentro, sendo informadas que iriam ver alguém conhecido que deveriam descrever. $\mathrm{O}$ objetivo foi que, ao abrir a caixa e ver o espelho, a gestante tivesse a oportunidade de refletir sobre suas potencialidades e 
fragilidades, propiciar reflexões e autoconhecimento.

O segundo encontro contou com a participação de sete gestantes, interna de enfermagem, nutricionista, assistente social e enfermeira da unidade. A assistente social abriu a temática de paternagem com um diálogo sobre a relevância do companheirismo e a ajuda mútua no cuidado da família para uma relação harmoniosa.

$\mathrm{O}$ terceiro encontro contou com a presença de oito gestantes, interna de enfermagem, nutricionista, assistente social, psicólogo e enfermeira da unidade, com explanação dos direitos trabalhistas das gestantes. Assim, abordaram-se os direitos que estão previstos na Consolidação das Leis do Trabalho (CLT), com abertura de diálogo compartilhado sobre licença maternidade; direito à dispensa do trabalho para, no mínimo, seis consultas pré-natal e exames complementares; direito a duas pausas de meia hora cada, para amamentar o bebê até os seis meses de idade; afastamento, enquanto durar a gestação e a lactação, de qualquer atividade ou local insalubre. O tema gerou muitos esclarecimentos, fortalecendo os conhecimentos dos direitos das gestantes em suas atividades laborais.

O tema sinais de risco na gestação foi discutido no quarto encontro, que teve como participantes a interna de enfermagem, enfermeira, psicólogo, nutricionista, assistente social, fisioterapeuta, 11 gestantes e um pai. Como forma de sondar os conhecimentos e dúvidas das gestantes, foi realizada uma dinâmica chamada "Mitos e Verdades", em que elas tiravam de dentro de uma caixa afirmações sobre os riscos na gestação. Ao fim, foi explanado sobre todos sinais de risco na gestação, frisando a necessidade de procurar imediatamente o CSF ou maternidade de referência nos fins de semana, diante dos sinais de risco dialogados.

$\mathrm{O}$ quinto encontro contou com a participação de 11 gestantes, a interna de enfermagem, dois ACS e uma técnica de enfermagem, além da fisioterapeuta, que facilitou uma estação prática com exercícios físicos que fortalecem a musculatura do assoalho pélvico.

No sexto encontro abordou-se a sexualidade na gestação por meio de uma roda de conversa, na qual seis gestantes participantes tiveram espaço para expor suas dúvidas e saberes, dialogar sobre a temática com a interna de enfermagem e a enfermeira da unidade e desmistificar mitos e tabus que envolvem a sexualidade na gestação.

O sétimo encontro de intervenção abordou as técnicas corretas de 
amamentação, bem como os benefícios para bebê e genitora. $\mathrm{O}$ mesmo contou com a participação de 11 gestantes, interna de enfermagem, enfermeira e nutricionista. Com auxilio de boneca e prótese mamária, explicaram-se as formas corretas de amamentação. Após mostrou-se um vídeo para que elas visualizassem a prática correta, frisando também na relevância do aleitamento materno exclusivo (AME) até os seis meses de vida do bebê. Cada gestante teve a oportunidade de praticar as técnicas ensinadas com o auxílio das gestantes que já tinham experiência.

No oitavo encontro de intervenção, realizou-se momento de lazer na piscina para fortalecimento de vínculos, que teve a participação de oito gestantes, a interna de enfermagem, enfermeira da unidade e dois ACS. Já no nono encontro de intervenção, realizou-se o Book Gestante com profissionais da beleza no próprio CSF, com serviços de maquiagem e cabelos gratuitamente, além de um cenário que foi construído para o registro fotográfico de 12 gestantes.

No décimo encontro realizou-se uma roda de conversa sobre os nomes dos bebês e cuidado na escolha. Participaram do encontro 10 gestantes, a interna de enfermagem, a enfermeira da unidade e dois
ACS. Cada gestante recebeu uma plaquinha com seu nome, informando a origem $\mathrm{e}$ significado, permeando uma discussão sobre a importância dos nomes, o significado e a importância que eles têm e, consequentemente, o cuidado que se deve ter na escolha. Além disso, foi entregue um papel para que as mesmas colocassem uma nota como forma de avaliação das intervenções. As quais atribuíram nota 10, explanando espontaneamente a relevância das temáticas trabalhadas, afirmando que se sentiram mais seguras frente à gestação e maternidade.

\section{DISCUSSÃO}

Durante o acompanhamento prénatal, a autoestima materna deve ser um aspecto avaliado, considerando que esta é a base para o primeiro vínculo entre mãe e filho. Vale ressaltar que a paternidade é uma temática que vem sofrendo modificações em seu conceito. Da figura clássica de provedor, passando a abarcar atitudes de maior envolvimento e contato afetivo com os filhos, o que possibilita a mudança de padrões tradicionais de cuidado ao bebê, alterando o significado nos relacionamentos familiares. $^{5}$

Também é muito importante que a gestante seja orientada sobre os sinais que 
podem significar risco para a gestação e, na presença de qualquer um deles, procurar o serviço de saúde e ser encaminhada para consulta médica com urgência. ${ }^{7}$

A informação é primordial quando se fala no respeito aos direitos, pois essa implica em capacitar as gestantes para agirem autonomamente, visto que a maioria possuía vínculo empregatício. Quando as mulheres estão cientes dos seus direitos, são mais exigentes, menos passivas, mais atuantes e empoderadas. Em Tocantins, encontros de educação em saúde com grupo de gestantes se mostrou uma ferramenta potente de capacitação sobre desenvolvimento da gestação, aleitamento materno e direitos da mulher grávida. ${ }^{8}$

Durante os encontros, percebeu-se que as gestantes possuíam medo de realizar atividades físicas, entretanto as mulheres grávidas não devem entrar num estado de confinamento, mas serem incentivadas a continuar suas atividades. As grávidas, ao manterem uma rotina de exercícios, têm seu peso controlado e se sentem melhor, tanto física quanto psicologicamente. ${ }^{9}$ Grupo de gestantes em Fortaleza foi considerado um espaço estratégico de cuidados e apoio às grávidas na atenção básica. ${ }^{10}$

Outra temática retratada foi expressão da sexualidade na gravidez, que é influenciada por aspectos anatômicos, fisiológicos e psicológicos, juntamente a mitos, tabus, questões religiosas e socioculturais. Por carência de orientações profissionais durante a consulta pré-natal, gestante e parceiro desenvolvem preconceitos sobre as práticas sexuais na gravidez, por sentirem medo de prejudicar o feto durante o coito e outros fatores que podem interferir negativamente na educação sexual. ${ }^{11} \mathrm{Na}$ Bahia, atividades educativas foram apontadas como estratégia para que as gestantes tenham um pré-natal qualificado, pois atividades educativas em grupo favorecem o compartilhamento das informações entre usuárias e profissionais de saúde. ${ }^{12}$

Para evitar o desmame precoce, realizou-se junto com as gestantes e os profissionais de saúde a conscientização quanto aos benefícios do AME para a saúde da criança e da mulher e suporte a prática da amamentação. ${ }^{13}$ Relato de experiência com grupos de gestantes de Minas Gerais apontou a relevância de atividades educativas para a promoção da educação em saúde, por permitir a troca de saberes entre os profissionais e os gestantes, sendo atribuições do enfermeiro a capacitação do indivíduo, o estímulo e a promoção ao autocuidado. $^{14}$ 
Nesse sentido, a abordagem educativa no contexto dos serviços de saúde é considerada eixo fundamental em que devem ser realizadas atividades de promoção da saúde, para além da assistência, a fim de apoiar o desenvolvimento social e pessoal por meio de informações educativas. A promoção da saúde consiste, assim, em capacitar a comunidade para atuar na melhoria de seu autocuidado e qualidade de vida. ${ }^{15}$

As atividades de educação em saúde com as gestantes permitiu a aproximação entre profissionais e receptores do cuidado, além de contribuir para uma assistência mais humana e qualificada. Vale observar a importância dos grupos de educação em saúde como instrumento de aquisição de conhecimentos para acadêmicos e profissionais, sobre manejo de situações clínicas na gestação, além de um espaço de ressignificação, reflexão de atitudes, condutas e papel profissional, mudanças nos sentimentos despertados pelo cuidado das gestantes, contribuindo na gênese de profissionais capacitados e humanizados, comprometidos com as necessidades de saúde da população.

Assim, o círculo de cultura se mostra como uma estratégia ativa de aprendizagem e estímulo à participação, ao promover a capacitação para o autocuidado e a busca por atitudes geradoras de mudanças. Por constituir metodologia participativa e inovadora, favorece a capacitação, com a possibilidade de decisão sobre seus próprios destinos, o que contribui para atuarem na melhoria de sua condição de saúde. ${ }^{16}$

\section{CONSIDERAÇÕES FINAIS}

As vivências educativas junto ao grupo de gestantes propiciaram encontros participativos e dialogados, com fortalecimento de vínculos entre os diferentes atores envolvidos no processo de gestação, com instrução sobre temáticas inerentes ao período gravídico e expansão de cuidados por meio do compartilhamento de saberes, permuta de experiências e esclarecimento de dúvidas.

Foi possível perceber uma boa adesão das gestantes ao grupo educativo no CSF, todavia é válido inferir que algumas delas residiam em área distante da unidade e/ou trabalhavam, o que dificultou a participação das mesmas em todos os encontros.

Ainda que o estudo apresente limitações, por se tratar da vivência de apenas um grupo, a experiência relatada propiciou troca de saberes entre a interna de enfermagem, equipe de saúde da familia, residentes multiprofissionais $\mathrm{e}$ 
gestantes, agregando melhorias ao processo de educação em saúde, baseado na construção compartilhada de conhecimentos, ampliando o espaço universitário e exercitando o compromisso social da Universidade.

\section{REFERÊNCIAS}

1. Macinko J, Harris MJ. Brazil's Family Health Strategy - delivering communitybased primary care in a universal health system. N Engl J Med 2015; 372:2177-81. 2. Nunes JT, Gomes KRO, Rodrigues MTP, Mascarenhas MDM. Qualidade da assistência pré-natal no Brasil: revisão de artigos publicados de 2005 a 2015. Cad. Saúde Colet., 2016; 24 (2): 252-261. 3. Barreto CN, Ressel LB, Santos CC, Wilhelm LA, Silva SC, Alves CN. Atenção pré-natal na voz das gestantes. Rev enferm UFPE on line., 2013;7(5):4354-63.

4. Souza VB, Roecker S, Marcon SS. Ações educativas durante a assistência pré-natal: percepção de gestantes atendidas na rede básica de Maringá-PR. Rev. Eletr. Enf. 2011; 13(2):199-210.

5. Fabbro MRC, Lima MP. A experiência paterna de pais de "primeira viagem" no cuidado do bebê nos primeiros três meses de vida. Atas CIAIQ2017. Investigação Qualitativa em Saúde. 2017; 2(1): 166-175. 6. Linhares FMP, Pontes CM, Osório MM. Construtos teóricos de Paulo Freire norteando as estratégias de promoção à amamentação. Rev. Bras. Saude Mater. Infant. 2014; 14(4):433-439.

7. Carvalho VF, Kerber NPC, Azambuja EP, Bueno FF, Barros AM. Direitos das parturientes: conhecimento da adolescente e acompanhante. Saúde Soc. 2014; (2): 572581.

8. Cunha MVB, Carneiro LS, Oliveira JD, Evangelista DR. Intervenção educativa para gestantes: avaliação do conhecimento. Rev Cereus. 2018; 10(3): 1-16.

9. Afonso ALM. Nível de consciência dos benefícios do exercício físico na gestação. [Trabalho de Conclusão de Curso]. Universidade Federal do Rio Grande do Norte. 2017. 32f. 10. Queiroz MVO, Menezes GMD, Silva TJP, Brasil EGM, Silva RM. Grupo de gestantes adolescentes: contribuições para o cuidado no pré-natal. Rev Gaúcha Enferm. 2016;37(esp):e2016-0029.

11. Jamali S, Mosalanejad L. Sexual dysfunction in Iranian pregnant women. Iran J Reprod Med. 2013; 11(6):479-86. 12. Silva ALS, Nascimento ER, Coelho EAC, Nunes IM. Atividades educativas no pré-natal sob o olhar de mulheres grávidas. Rev Cubana de Enfer. 2014; 30(1):1-15. 13. Coca KP, Pinto VL, Westphala F, Mania PNA, Abrão ACFV. Conjunto de medidas para o incentivo do aleitamento materno exclusivo intra-hospitalar: evidências de revisões sistemáticas. Rev Paul Pediatr. 2018; 36(2):214-220.

14. Neves AM, Mendes LC, Silva SR. Práticas educativas com gestantes adolescentes visando a promoção, proteção e prevenção em saúde. Rev Min Enferm. 2015; 19(1): 241-244.

15. Ministério da Saúde (BR), Secretaria de Gestão Estratégica e Participativa. II Caderno de educação popular em saúde. Brasilia: Ministério da Saúde; 2014. 16. Machado ALG, Borges FM, Silva AZ, Jesus ACP, Moreira TMM, Cunha NF. Círculo de cultura na promoção da saúde de idosos hipertensos: relato de experiência. Cienc Cuid Saude. 2017; 16(1):1-6.

RECEBIDO: $22 / 12 / 2017$

APROVADO: $28 / 06 / 2019$

PUBLICADO: 07/2020 